\title{
Dengue vector surveillance methods in Muntinlupa City, Philippines
}

\author{
Jennifer Duncombe ${ }^{a}$ and Kristian Marollano ${ }^{b}$ \\ Correspondence to Jennifer Duncombe (e-mail: j.duncombe@uq.edu.au)
}

We read with great interest the article by Chang et al. ${ }^{1}$ about the future of dengue vector control in the Western Pacific Region. We are currently undertaking Aedes monitoring in San Jose Village, Muntinlupa City, Philippines, where we seek to determine the most sustainable dengue vector surveillance method for Muntinlupa City. Our study involves comparing data from fortnightly collections of adult Aedes mosquitoes in sticky Ovitraps with a one-off pupal survey conducted in the same area. We will also determine the prevalence of Aedes aegypti and Aedes albopictus in the village and investigate the role of spatial heterogeneity in vector surveillance. Previous pupal surveys have shown mixed results for Aedes density in San Jose Village and were very resource-intensive, which limited their sustainability.

This work is part of a larger study that aims to build a low-cost, geographically-enhanced dengue data management system for use by local health authorities. The system will collate data from a range of sources and produce regular reports and maps showing cases, vectors and predicted dengue clusters. Local health authorities can use these outputs to better target dengue control activities, including community education, removal of breeding sites, preventive fogging and improved waste and water management.

San Jose Village is a gated community of 203 households with low population density $\left(2.48\right.$ per $\left.1000 \mathrm{~m}^{2}\right)$. The environment is semi-rural, with abundant vegetation; the weather is equatorial: hot and humid, with a wet and dry season each year. During the wet season, dengue cases increase dramatically due to the abundant rainfall and consequent increase in Aedes breeding sites. Fifty sticky Ovitraps were donated to the study by the Queensland Tropical Health Unit, Australia. The sticky Ovitraps are relatively inexpensive and easily constructed. They are designed to attract gravid adult female Aedes mosquitoes; when they fly into the trap they get stuck on the polybutylene adhesive panel inside. ${ }^{2}$ To avoid the traps becoming a breeding site for Aedes mosquitoes, they are checked for the presence of pupae/larvae and water is changed weekly.

Since the sticky Ovitraps were deployed in the village in June 2011, they have been well received and maintained by householders. We have noticed that householders have been more receptive to keeping the traps on their property compared with previous years. This may be because they recognize the study team, are used to being asked to participate in dengue studies and see the value of the research.

Due to the relative ease of implementing and monitoring the sticky Ovitraps and receptiveness of the community in this pilot study, it is anticipated that sticky Ovitraps will become a sustainable vector monitoring tool in Muntinlupa City, Philippines. Consistent with Chang et al., we support the improvement of vector surveillance in the Western Pacific Region and agree that Geographical Information Systems (GIS) should be used to better target dengue control and ultimately reduce disease transmission and prevalence.

\section{Conflicts of interest}

None declared.

\section{References:}

1. Chang MS et al. Challenges and future perspective for dengue vector control in the Western Pacific Region. Western Pacific Surveillance and Response Journal, 2011, 2(2):9-16. doi:10.5365/wpsar.2010.1.1.012.

2. Ritchie SA et al. An adulticidal sticky ovitrap for sampling container-breeding mosquitoes. Journal of the American Mosquito Control Association, 2003, 19:235-242. pmid: 14524545

\footnotetext{
a School of Population Health, University of Queensland, Australia

a Department of Medical Entomology, Research Institute for Tropical Medicine, the Philippines

Submission date: 1 August 2011; Publication date: 17 August 2011

doi: 10.5365/wpsar.2011.2.3.001
} 